\title{
The Stiffness of the Blood Vessels as a Component of Cardionephro - Cerebrovascular Risk for Obesity
}

\author{
Murkamilov Ilham Torobekovich ${ }^{1,2 *}$ and VV Fomin ${ }^{3}$ \\ ${ }^{1}$ I.K. Akhunbaev Kyrgyz State Medical Academy, 92 Akhunbaev str., Bishkek 720020, Kyrgyzstan \\ ${ }^{2}$ First President of Russia B. N. Yeltsin Kyrgyz Russian Slavic University, 44 Kievskaya str., Bishkek 720000, Kyrgyzstan
}

${ }^{3}$ I.M. Sechenov First Moscow State Medical University, 2 Bolshaya Pirogovskaya str., Moscow 119991

*Corresponding author: Murkamilov Ilham Torobekovich, Kyrgyz State Medical Academy Akhunbaeva and Kyrgyz-Russian Slavic University, Kyrgyzstan, Email: murkamilov.i@mail.ru

To Cite This Article: Murkamilov Ilham Torobekovich. The Stiffness of the Blood Vessels as a Component of Cardionephro - Cerebrovascular Risk for Obesity. Am J Biomed Sci \& Res. 2019 - 4(2). AJBSR.MS.ID.000763. DOI: 10.34297/AJBSR.2019.04.000763

Received: June 24, 2019 | Published: July 18, 2019

\begin{abstract}
Summary: In the studied sample the prevalence of 1st degree obesity was 70.4\%. In 1st degree obesity, a significant increase in the stiffness index and the duration of the pulse wave are recorded, which were significantly more often associated with the type of the "A" pulse curve. In 3rd degree obesity, a significant increase in the level of central aortic and systolic blood pressure was accompanied by a statistically significant increase in the level of augmentation index and the value of augmentation index brought to a pulse of 75 beats per minute. In persons with obesity, a relationship was noted between the value of body mass index with a stiffness index, an alternative stiffness index, and A2d4 vascular parameters.
\end{abstract}

Materials and Methods: The study included 715 people aged 18 to 88 years (mean age $53.5 \pm 11.8$ years) with obesity, women 409 (57.2\%) and men 306 (42.8\%). All examined individuals were measured for body length $(\mathrm{cm})$, body weight $(\mathrm{kg})$ with the determination of body mass index (BMI, $\mathrm{kg} / \mathrm{m} 2$ ), blood pressure (BP) systolic and diastolic ( $\mathrm{mm} \mathrm{Hg}$ ), frequency heart rate (HR, beats/min). Depending on the BMI classified obesity (WHO, 1997): 1st degrees (30-34.9 kg/m2), 2nd degree (35-39.9 kg/m2), 3rd degree (more than $40 \mathrm{~kg} / \mathrm{m} 2$ ). Investigated the central arterial (aortic) pressure (CAP) and the parameters of vascular stiffness (VSt) on the apparatus "AngioScan-01" (LLC Angioscan-Electronics, Russia).

Results: The presence of grade 1 obesity was detected in $70.4 \%$ of the examined individuals, grade 2 obesity in $22.3 \%$, grade 3 obesity in $7.1 \%$. In the studied sample, people of middle (45.1\%) and elderly (27.8\%) age prevailed. Among the study participants, obesity in $10.2 \%$ and $46.5 \%$ of cases was associated with smoking and tachycardia. Significant increase in central aortic pressure $(140 \pm 20 \mathrm{mmHg}$. versus $133 \pm 21 \mathrm{mmHg}$; $<0.05)$ and systolic blood pressure $(143 \pm 20 \mathrm{mmHg}$. vs. $135 \pm 20 \mathrm{mmHg}$; $<0.05)$ were observed in patients with obesity of the 3rd degree compared with the 1st degree.

A statistically significant increase in the augmentation index and the value of the augmentation index resulted in a pulse rate of 75 beats per minute (AIp75) of the vessels was recorded in persons with grade 3 obesity. The stiffness index and the duration of the pulse wave were significantly higher in the subgroup of examined individuals with 1st degree obesity. The type of pulse curve " $\mathrm{A}$ " was more often detected in persons with obesity grade 1. A direct link is noted between the value of BMI with a stiffness index $(r=0.298 ; p=0.001)$, an alternative stiffness index ( $r=0.119 ; p=$ $0.001)$ and $\mathrm{A} 2 \mathrm{~d} 4$ indicators $(\mathrm{r}=0.101 ; \mathrm{p}=0.001)$ of the vessels. In patients with obesity, the most associated diseases were arterial hypertension (64.3\%), comorbid diseases (34.8\%) and chronic obstructive pulmonary disease (14.4\%). In addition, among those examined with obesity, cerebrovascular diseases were detected in 3.3\%, coronary heart disease in $8.5 \%$, chronic kidney disease in $9.9 \%$, and type 2 diabetes in $11.3 \%$.

Keywords: Obesity; Vascular Stiffness; Pulse Wave Velocity; Risk Factors; Cardiovascular Diseases

\section{Introduction}

The role of obesity in the development of cardiovascular diseases (CVD) is currently widely discussed. According to WHO, about 1.4 billion adults in the world are overweight, and over 500 million people are obese [1,2]. Among the possible mechanisms of the occurrence of CVD in people with obesity, it is customary to consider an increase in vascular stiffness (VSt). It has been shown that increased in VSt is associated with the aging process and is often combined with high sodium intake, arterial hypertension $(\mathrm{AH})$, and diabetes mellitus. (DM), hyper- and dyslipidemia, obesity, and neurohormonal activation of the sympathetic nervous system 
(SNS) [3-5]. These factors lead to endothelial dysfunction, vascular inflammation, hyperplasia of vascular smooth muscle cells, increased degradation of collagen and elastin [6].

The increase in VSt is a consequence of fibrosis and degradation of the elastic fibers of large arteries. Increased arterial stiffness leads to the development of hypertension $[7,8]$. So, the famous clinician of the 20th century E.M. Tareev in 1948 noted that "The concept of hypertension is most often associated with obese hypersthene's, with a possible violation of protein metabolism, with blood clogged with products of incomplete metamorphosis cholesterol, uric acid ..." [9] Information on the problem of obesity, as a rule, was obtained in large, epidemiological studies $[10,11]$. Currently, people with obesity is of importance to the assessment of the mechanical properties of blood vessels: stiffness, extensibility and compliance of their walls. However, in the literature there are few works devoted to the analysis of VSt in obesity, considering its degree. Purpose of the study. To study and analyze the state of vascular stiffness and central hemodynamics in obesity.

\section{Materials and Methods}

In total, for the period from September 2016 to February 1, 2018, 715 patients were included: 409 (57.2\%) of them were women and $306(42.8 \%)$ of men aged from 18 to 88 years old (average age $53.5 \pm 11.8$ years). The presence and degree of obesity was determined according to the WHO classification in terms of body mass index (BMI, $\mathrm{kg} / \mathrm{m} 2$ ). In all the examined individuals, body length $(\mathrm{cm})$ and body weight $(\mathrm{kg})$, blood pressure (BP) were measured systolic and diastolic $(\mathrm{mm} \mathrm{Hg})$, frequency heart rate (HR, beats/min). Depending on BMI, classified obesity (WHO, 1997): 1st degree (30-34.9 kg / m2), 2nd degree (35-39.9 kg/m2), 3rd degree (more than $40 \mathrm{~kg} / \mathrm{m} 2$ ).

Studies of the central arterial (aortic) pressure (CAP) and the parameters of vascular stiffness (VSt) were performed on the Angioscan-01 device (Angioscan-Electronics LLC, Russia) [12]. The augmentation index (AI, Alp, augmentation index) was calculated as the difference between the second and first systolic pressure peaks of the pulse wave, which were expressed as a percentage of the pulse pressure. Pressure augmentation called the difference between pressure of the first (T1) and second (T2) systolic peak of the pulse wave. It is this part of pulse blood pressure that increases significantly with an increase in VSt, causing the growth of CVD and an increase in the afterload on the left ventricular (LV) myocardium.

The value of the augmentation index was expressed in percent and was calculated using the formula: AIp $=100 \% *(\mathrm{D}$ [T2] - D [T1]) / D [Tmax], where D [Tn] is the data value at time n. In addition, all participants studied the type of pulse curve. Allocated the following types. "C" type of curve is characterized by finding the maximum of the reflected wave (RW) in the late systolic phase and its projection point on the original curve (yellow color) is located on the descending arm close to the moment of aortic valve collapse. This type of curve is observed in young people under 30 years of age who do not have risk factors for the development of CVD associated with atherosclerosis. The type " $\mathrm{B}$ " of the curve is characterized by the finding of the maximum of the RW in the middle of the systole, and the point of projection on the initial curve almost coincides with the global maximum of the initial curve. This type of curve is observed in people older than 40 years, or in younger, but with risk factors for the development of CVD.

The " $\mathrm{A}$ " curve type is characterized by the coincidence of the global maximum of the original signal curve with the maximum point of the late systolic wave. This type of curve is observed in elderly people or when a combination of high rigidity of large conducting arteries and high tone of small resistive arteries. Statistical analysis of the data was carried out using the software "Statistica 10.0". All quantitative variables are presented as mean \pm standard deviation, medians and quartiles [Me (Q25; Q75)]. Significance of differences between groups was assessed using t-criterion Student's tutorial (for variables with a normal distribution) and the Mann-Whitney test (for variables with a nonparametric distribution) [13]. Correlation analysis was carried out by the Pearson criterion - for variables with a normal distribution and the Spearman coefficient (for variables with a non-parametric distribution). For all types of analysis, $\mathrm{p}<0.05$ were considered statistically significant.

\section{Research Results}

Table 1: HR-heart rate; $n$ - is the number of patients.

\begin{tabular}{|c|c|c|c|c|}
\hline Indicators & $n=715$ Total & $n=409$ Women & $n=306$ Men & $\mathbf{P}$ \\
\hline Obesity 1st degree, n (\%) & $504(70,4)$ & $279(68,2)$ & $225(73,5)$ & 0,148 \\
\hline Obesity 2nd degree, n (\%) & $160(22,3)$ & $96(23,4)$ & $64(20,9)$ & 0,523 \\
\hline Obesity grade 3, n (\%) & $51(7,1)$ & $34(8,3)$ & $17(5,5)$ & 0,057 \\
\hline Young age, n (\%) & $12(1,6)$ & $5(1,2)$ & $7(2,2)$ & 0,059 \\
\hline Mature age, n (\%) & $154(21,5)$ & $73(17,8)$ & $81(26,4)$ & 0,025 \\
\hline Mean age, $\mathrm{n}(\%)$ & $323(45,1)$ & $193(47,1)$ & $130(42,4)$ & 0,183 \\
\hline Older age, n (\%) & $199(27,8)$ & $125(30,5)$ & $74(24,1)$ & 0,039 \\
\hline Senile age, n (\%) & $27(3,7)$ & $13(3,1)$ & $14(4,5)$ & 0,165 \\
\hline Smoking, n (\%) & $73(10,2)$ & $6(1,7)$ & $67(21,8)$ & 0,005 \\
\hline $\mathrm{HR}>80$ beats / $\min , \mathrm{n}(\%)$ & $333(46,5)$ & $182(44,4)$ & $151(49,3)$ & 0,184 \\
\hline
\end{tabular}


The distribution of study participants by degree of obesity and by age are presented in Table 1 . In our study, the proportion of people with obesity grade 1 , both in the total sample and in women and men, was numerous. Among the surveyed, people of middle (45.1\%) and elderly (27.8\%) age prevailed. In our study, obesity in $10.2 \%$ and $46.5 \%$ of cases was associated with smoking and tachycardia, respectively. Morbid obesity was detected in 51 individuals in $7.1 \%$ of cases. The number of participants surveyed with obese adulthood and the frequency of smoking was significantly higher among males. As can be seen from the obtained data, the proportion of elderly people with obesity significantly prevailed in women (Table 1).

\section{Clinical Characteristics of Obese People Examined}

Conducting a correlation analysis allowed us to detect the effect of obesity on the increase in VSt. A direct relationship is noted between the value of BMI with the stiffness index ( $r=0.298$; $p=$ 0.001), the alternative stiffness index $(r=0.119 ; p=0.001)$ and A2d4 ( $r=0.101 ; p=0.001)$ VSt. Table 3 shows that the correlation analysis did not reveal changes in VSt indices (AIP, AIP 75, AGI, VA, RI and PWA) depending on the level of BMI.

\section{The Prevalence of Nosological types of Socially Significant Diseases Obese People Examined $\mathbf{n}=715$}

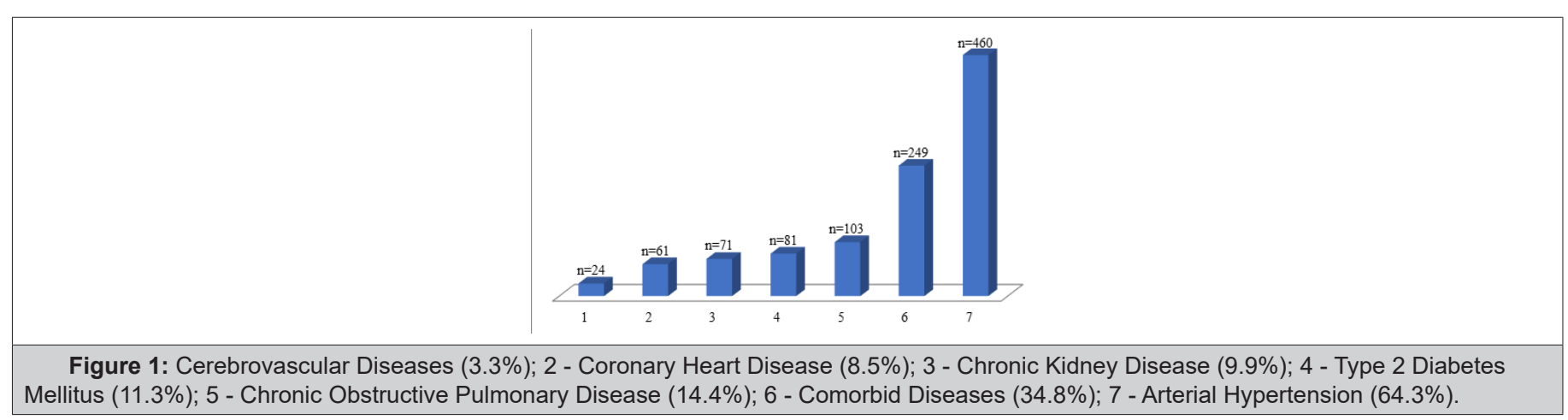

Presented in Figure 1 data indicate that in patients with obesity, the most associated nosologies were hypertension (64.3\%) and comorbid diseases (34.8\%). In addition, in $14.4 \%$ of cases, obesity was combined with chronic obstructive pulmonary disease (COPD), $3.3 \%$ - cerebrovascular disease, $8.5 \%$ - coronary heart disease (CHD), $11.3 \%$ - diabetes mellitus 2 nd type and 9.9\% - chronic kidney disease. The prevalence of nosological types of socially significant diseases obese people examined $n=715$ (Figure 1).

\section{Discussion}

Obesity is a serious world-class medical and social problem, and its combined course with hypertension, DM, COPD and CHD is characterized by a decrease in the quality of life, increase in the frequency and duration of hospitalization [14,15]. The present study summarizes the current views on the mechanisms of formation of cardio-nephritic and cerebrovascular risk in obesity with an emphasis on VSt. An important role in the regulation of SJ in people with obesity is played by the renin-angiotensin-aldosterone system (RAAS) [16,17]. Experimental studies have shown that an increase in arterial stiffness is associated with structural changes in the vascular wall $[17,18]$. This is manifested by diffuse intimal fibroelastic thickening with endothelial remodeling, changes in the extracellular matrix, increased content and disorganization of collagen, fragmentation of the elastic membrane, infiltration of walls with smooth myocytes, fibrosis and calcification [19]. Many studies have found that adipokines of adipose tissue contribute to the development of a sluggish inflammatory process in people with obesity, leading to a complex of metabolic disorders, cardiovascular complications (CVC) and to autoimmune inflammatory diseases $[14,15]$.

\begin{tabular}{|c|c|c|}
\hline Table 2: Correlation Analysis Between Body Mass Index and Vascular Stiffness Parameters in Obese Individuals Examined \\
\hline \multirow{2}{*}{ Indicators } & \multicolumn{2}{|c|}{ Indicators of BMI, kg/m2 } \\
\cline { 2 - 3 } & $\mathbf{r}=$ & $\mathbf{p}=$ \\
\hline Stiffness index (SI), \% & 0,298 & 0,001 \\
\hline Alternative stiffness index (aSI), \% & 0,119 & 0,001 \\
\hline The augmentation index (AIP), \% & 0,050 & 0,180 \\
\hline The augmentation index reduced to pulse 75 (AIP 75) & 0,050 & 0,176 \\
\hline Age Index (AGI) & 0,058 & 0,122 \\
\hline The average age of the vascular system (VA) & 0,057 & 0,761 \\
\hline Reflection Index (RI) & 0,011 & 0,166 \\
\hline Pulse wave amplitude (PWA) & 0,041 & 0,007 \\
\hline A2d4 & 0,101 & \\
\hline
\end{tabular}

Note: BMI=body mass index; $\mathrm{n}$ is the number of patients; $\mathrm{p}$ - reliability. 
Systemicinflammation and adipokine production are considered as the most important mechanisms by which adipose tissue has a damaging effect on the vascular wall [20]. The content of one of adipocytokines, leptin, is increased in obesity and is associated with MTR, regardless of BMI and traditional cardiovascular risk factors [21]. Several researchers indicate a weak and moderate correlation of VSt parameters with BMI [22]. This confirms the well-known fact that under the influence of BMI and hypercholesterolemia, atherosclerotic processes in the vessels are more pronounced, which contributes to their remodeling [23]. It has been shown that an increase in aortic stiffness, assessed by the degree of increase in the pulse wave velocity (SPV), is an independent predictor of the risk of CVC [24]. It is considered that SPV is a parameter that integrates the geometry and elastic properties of blood vessels. It should be noted that the close relationship between the magnitude of the BMI and the indirect signs of SST obtained in this study (Table 2).

\begin{tabular}{|c|c|c|c|}
\hline Indicators & $\begin{array}{c}\text { Obesity } 1^{\text {st }} \text { degree } \\
n=504\end{array}$ & $\begin{array}{c}\text { Obesity } 2^{\text {nd }} \text { degree } \\
n=160\end{array}$ & $\begin{array}{c}\text { Obesity } 3^{\text {rd degree }} \\
n=51\end{array}$ \\
\hline Sex, women / husband & $279 / 225$ & $96 / 64$ & $34 / 17$ \\
\hline Age years & $53,5 \pm 12,2$ & $53,1 \pm 10,9$ & $53,2 \pm 11,1$ \\
\hline BMI, $\mathrm{kg} / \mathrm{m} 2$ & $31,9 \pm 1,3$ & $36,7 \pm 1,3$ & $43,3 \pm 3,1$ \\
\hline HR, beats / min & $79 \pm 13$ & $80 \pm 12$ & $83 \pm 17$ \\
\hline Systolic blood pressure, mm Hg Art. & $133 \pm 21$ & $134 \pm 19$ & $140 \pm 20^{*}$ \\
\hline TsAP, mm Hg Art. & $135 \pm 20$ & $136 \pm 19$ & $143 \pm 20^{*}$ \\
\hline Diastolic blood pressure, mm Hg st. & $86 \pm 10$ & $89 \pm 11$ & $90 \pm 10$ \\
\hline Young age, n (\%) & $9(1,7)$ & $2(1,2)$ & $1(1,9)$ \\
\hline Mature age, n (\%) & $107(21,2)$ & $36(22,5)$ & $11(21,5)$ \\
\hline Mean age, n (\%) & $226(44,8)$ & $76(47,5)$ & $21(41,1)$ \\
\hline Older age, n (\%) & $141(27,9)^{* * *}$ & $41(25,6)$ & $1(1,9)$ \\
\hline Senile age, n (\%) & $21(4,1)$ & $5(3,1)$ & $17(33,3)^{* *}$ \\
\hline SI (Stiffness Index), m/s & $7,73 \pm 0,90^{* * *}$ & $7,64 \pm 0,81$ & $7,30 \pm 1,60$ \\
\hline $\mathrm{aSI}, \mathrm{m} / \mathrm{s}$ & $9,19 \pm 2,79$ & $9,46 \pm 2,90$ & $8,95 \pm 3,5$ \\
\hline AIP, $\%$ & $11,5(1,40 ; 22,2)$ & $9,7(-1,70 ; 22,1)$ & $13,0(-6,40 ; 24,8)^{* *}$ \\
\hline AIP 75, \% & $14,2(5,10 ; 22,7)$ & $12,6(2,7 ; 23,5)$ & $14,9(2,40 ; 27,1)^{* *}$ \\
\hline AGI, (Aging Index) & $-0,4(-0,7 ;-0,2)$ & $-0,4(-0,70 ;-0,20)$ & $-0,5(-0,70 ;-0,30)$ \\
\hline VA, years old & $53,7 \pm 15,5$ & $55,5 \pm 13,9$ & $54,4 \pm 14,4$ \\
\hline RI, (Reflection Index) \% & $40,8 \pm 18,7$ & $40,4 \pm 17,4$ & $41,6 \pm 19,4$ \\
\hline Sat02 & $94,4 \pm 4,8$ & $93,8 \pm 3,5$ & $93,3 \pm 3,0$ \\
\hline PWA, & $2,81 \pm 0,75$ & $2,79 \pm 0,97$ & $2,78 \pm 0,70$ \\
\hline PD, (Pulse Duration) мс & $770,5 \pm 126,3^{* * *}$ & $770,2 \pm 115,0$ & $756,8 \pm 170,9$ \\
\hline ED, (Ejection Duration) мс & $272,5 \pm 24,2$ & $272,3 \pm 24,5$ & $274,2 \pm 23,0$ \\
\hline T1, (Time to 1st Peak) мс & $107,8 \pm 13,0$ & $108,9 \pm 13,4$ & $108,4 \pm 14,0$ \\
\hline T2, (Time to 2nd Peak) мс & $203,0 \pm 21,4$ & $202,4 \pm 23,1$ & $202,6 \pm 23,1$ \\
\hline dTpp, мс & $95,2 \pm 20,6$ & $93,5 \pm 20,2$ & $94,2 \pm 23,4$ \\
\hline \multicolumn{4}{|c|}{ The Distribution of the Types of Pulse Curve in the Examined Subgroups } \\
\hline A type & $437(86,7)^{* * *}$ & $70(43,7)$ & $25(49,0)$ \\
\hline In type & $31(6,1)$ & - & - \\
\hline With type & $36(7,1)$ & $9(5,6)$ & $4(7,8)$ \\
\hline B + C type & $30(5,9)$ & $13(8,1)$ & $6(11,7)^{*}$ \\
\hline A + B type & $80(15,8)$ & $19(11,8)$ & $2(3,9)^{*}$ \\
\hline A + B + C type & $124(24,6)$ & $40(25)$ & $14(27,4)$ \\
\hline
\end{tabular}

BMI - body mass index; HR - heart rate; TsAP - central arterial (aortic) pressure; SI - stiffness index; aSI - alternate stiffness index; AGI - age index; Alp75 - the value of the augmentation index resulted in a pulse of 75 beats per minute; VA - the average age of the vascular system; RI - vascular reflection index; AIP - augmentation index; SatO2 - peripheral blood oxygen saturation; PWA - pulse wave amplitude; PD is the pulse wave duration; ED - systole duration; T1 is the time interval from the beginning of the pulse wave to the maximum of the early systolic wave (direct wave); T2 - time indicator from the beginning of the pulse wave to the maximum point of the late systolic wave; dTpp- is a time parameter that determines the time between the maxima of the direct (early systolic) and reflected (late systolic) waves; $n$ is the number of patients; * $p<0.05$ between the 1 st; ${ }^{* *} p<0.05$ between the $2 n d ;{ }^{* * *} p<0.05$ between the $3 r d$. 
The prognostic value of SST in the arteries is most important even during the formation and at the initial stage of hypertension [25]. In some studies, it has been noted that in obesity, an increase in stiffness of the arteries and blood pressure is observed in comparison with patients with normal weight [26,27]. As noted, obesity decreases compliance and increases VSt. An increase in PWV is a sign of subclinical coronary atherosclerosis, which makes it possible to use this sign as an independent risk factor for $\mathrm{AH}$, the detection of which is especially important for obese patients who are asymptomatic. It is important to note that the PWV and the associated arterial augmentation index are interesting from the point of view of pathophysiology and have a great prognostic value. The role of SST as an independent risk factor for cardiovascular and renal diseases was summarized in our previous study [28,29]. We were able to demonstrate a significant increase in vascular augmentation index in persons with 3rd degree obesity, and persons with 1st degree obesity significantly increased the vascular stiffness index (Table 3).

There is evidence of a positive relationship between BMI and stiffness of the great vessels, which was evaluated directly by SPV [30]. With an increase in VSt, the pulse wave increases, the reflected wave returns earlier during systole, which is manifested by an increase in systolic and pulse BP and LV afterload [31]. Thus, the higher the stiffness of the aorta, the worse the subendocardial blood flow, which in turn leads to increased subendocardial myocardial ischemia [32]. As noted, with the spread of obesity, severe somatic diseases associated with it, such as hypertension, diabetes, COPD, CHD and others, are increasing and aggravated. Our data also showed that AH (64.3\%) and comorbid pathologies (34.8\%) were the most associated with obesity. The pathophysiological basis for the occurrence of hypertension in obesity is compensatory hyperinsulinemia, which develops due to insulin resistance, characteristic of all forms of obesity [14,15].

First, hyperinsulinemia increases the reabsorption of sodium in the proximal tubules of the kidneys, which leads to hypervolemia and an increase in the content of sodium and calcium in the walls of blood vessels, causing their spasm and increase in total peripheral vascular resistance (RPV) [14,15]. Secondly, it stimulates the utilization of glucose, amino acids, regulates expression, etc. Through mitogen-activated protein kinase, insulin promotes damaging vascular effects by stimulating various growth factors, which leads to the proliferation and migration of smooth muscle cells, proliferation of vascular wall fibroblasts, and the accumulation of extracellular matrix.

This leads to a narrowing of blood vessels and further increases the round focal length [33]. Third, hyperinsulinemia increases the activity of the CNS. In our study (Table 1) among the examined individuals with obesity in $46.5 \%$ of cases was associated with tachycardia. The role of hyperactivation of the SNA is a key mechanism of regulation of blood pressure and heart rate, and with an increase in its tone, cardiac output increases, vascular spasm occurs, and total peripheral vascular resistance increases, were noted in earlier studies [34]. Thus, all these processes cause remodeling of the cardiovascular system, leading to a loss of elasticity of the vascular wall, impaired microcirculation, increased general peripheral resistance, reduced renal blood flow and ultimately increased vascular resistance and the formation of hypertension, renal dysfunction, etc. [35].

\section{Conclusion}

The results showed that the prevalence of obesity 1st degree was $70.4 \%$. In persons with obesity 1 st degree, a significant increase in the vascular stiffness index and the duration of the pulse wave was found. Higher levels of central aortic and systolic blood pressure were observed in the subgroup of people with obesity grade 3 and were accompanied by a statistically significant increase in the level of augmentation index and the value of augmentation index brought to pulse 75 beats per minute. Obese individuals have been found to be addicted. between body mass index and stiffness index, alternative stiffness index and $\mathrm{A} 2 \mathrm{~d} 4$ vessel index.

\section{References}

1. (2019) WHO Obesity and overweight. World Health Organization 2015. Switzerland.

2. Koliaki C, Liatis S, Kokkinos A (2019) Obesity and cardiovascular disease: revisiting an old relationship Metabolism 92: 98-107.

3. Hudson L, Kinra S, Wong I, Deanfield J, Viner R, et al. (2017) Is arterial stiffening associated with adiposity, severity of obesity and other contemporary cardiometabolic markers in a community sample of adolescents with obesity in the UK? BMJ pediatrics open 1(1): e000061.

4. García-Prieto CF, Gil-Ortega M, Vega-Martín E, Rubio MA, Somoza B et al. (2019) Beneficial effect of bariatric surgery on abnormal MMP-9 and AMPK activities: potential markers of obesity-related CV risk. Frontiers in physiology 10(8): 553.

5. Bobkova IN, Gussaova SS, Stavrovskaya EV, Struve AV (2018) Nephrological aspects of surgical weight correction in morbid obesity. Ter Arkh 90 (6): 98-104.

6. Lakatta EG, Levy D (2003) Arterial and cardiac aging: major shareholders in cardiovascular disease enterprises: Part I: aging arteries: a "set up" for vascular disease. Circulation 107(1): 139-146.

7. Ecobici M, Stoicescu C (2017) Arterial Stiffness and Hypertension-Which Comes First? Maedica (Buchar) 12(3): 184-190.

8. Gade JL, Stålhand J, Thore CJ (2019) An in vivo parameter identification method for arteries: numerical validation for the human abdominal aorta. Comput Methods Biomech Biomed Engin 22(4): 426-441.

9. Sinitsina EN, Markovskiy VB, Galanova AS, Avshalumov AS, Shilov AM (2008) Obesity and arterial hypertension. Therapist 2: 35-45.

10. Whitlock G, Lewington S, Sherliker P, Collins R, Peto R, et al. (2009) Prospective Studies Collaboration. Bodymass index and cause-specific mortality in 900000 adults: collaborative analyses of prospective studies. Lancet 373(9669): 1083-1096.

11. Wang R, Zhang P, Gao C, Yu Y, Li B, et al. (2016) Prevalence of overweight and obesity and some associated factors among adult residents of northeast China: a cross-sectional study. BMJ open 6(7): e010828.

12. Parfenov AS (2012) Early diagnosis of cardiovascular diseases using hardware-software complex Angioscan -01. Poliklinika 2(1): 70-74.

13. Grinhalh T (2004) The basics of evidence-based medicine. Geotar-Med pp. 240.

14. Dedov II, Romantsova TI, Shestakova MV (2018) Rational approach to patients' treatment with type 2 diabetes and obesity: results of the All-Russian observational program. AURORA Obesity and metabolism 4(15): 48-58. 
15. Dedov II, Shestakova MV, Vikulova OK (2017) Epidemiology of diabetes mellitus in Russian Federation: clinical and statistical report according to the federal diabetes registry. Diabetes mellitus 20(1): 13-41.

16. Mahmud A, Feely J (2004) Arterial stiffness and the renin-angiotensinaldosterone system. J Renin-Angiotensin-Aldosterone Sys 5(3): 102-108.

17. Jia G, Aroor AR, Hill MA, Sowers JR (2018) Role of Renin-Angiotensinaldosterone system activation in promoting cardiovascular fibrosis and stiffness. Hypertension 72(3): 537-548.

18. Peng F, Pan H, Wang B, Lin J, Niu W (2015) The impact of angiotensin receptor blockers on arterial stiffness: a meta-analysis. Hypertens Res 38: 613-620.

19. Orlova YA, Ageev AF (2006) Arterial stiffness as an integral indicator of the cardiovascular bed: physiology, assessment methods and drug correction. A heart 2: 65-69.

20. Berg AH, Scherer PE (2005) Adipose tissue, inflammation, and cardiovascular disease. Circ Res 96: 939-949.

21. Iljina AE, Barskova VG, Nasonov EL (2009) Gout, hyperuricemia and cardiovascular risk. Rheumatology Science and Practice 47(1): 56-62.

22. Tomiyama H, Yamashina A, Arai T, Doba N, Hinohara S, et al. (2003) Influences of age and gender on results of noninvasive brachialankle pulse wave velocity measurement - a survey of 12517 subjects. Atherosclerosis 166(2): 303-309.

23. Chatzizisis YS, Coskun AU, Jonas M, Feldman CL, Stone PH, et al. (2007) Role of endothelial shear stress in the natural history of coronary atherosclerosis and vascular remodeling: molecular, cellular, and vascular behavior. J Am Coll Cardio 49(25): 2379-2393.

24. Ben-Shlomo Y, Spears M, Boustred C, Cockcroft JR, Wilkinson IB, et al. (2014) Aortic pulse wave velocity improves cardiovascular event prediction: an individual participant meta-analysis of prospective observational data from 17,635 subjects. J Am Coll Cardiac 63(7): 636646.

25. Bolotova NV, Posokhova NV, Dronova EG (2015) The risk of hypertension in children with metabolic syndrome in the age aspect. Lechaschi Vrach Journal 1: 32-35.
26. Urbina EM, Kimball Thomas R, Khouryet Philip R, Daniels SR, Dolan LM, et al. (2018) Increased arterial stiffness is found in adolescents with obesity or obesity-related type 2 diabetes mellitus. J Hypertens 28(8): 1692-1698.

27. Çelik A, Özçetin M, Yerli Y (2011) Increased aortic pulse wave velocity in obese children. Archives of the Turkish Society of Cardiology 39(7): 557-562.

28. Murkamilov IT, Aitbaev KA, Yusupov FA (2017) Pulse wave velocity as a novel risk factor for chronic kidney disease progression. Cardiovascular Therapy and Prevention 16(4): 83-87.

29. Murkamilov IT, Aitbaev KA, Fomin VV, et al. (2018) Blood plasma cystatin $\mathrm{C}$ level and its relationship with the augmentation index and central arterial pressure in therapeutic patients. Clinical nephrology (8)3: 31-40

30. Sakuragi S, Abhayaratna K, Gravenmaker Karen J, Telford RD, Abhayaratna WP et al. (2009) Influence of adiposity and physical activity on arterial stiffness in healthy children: The Lifestyle of Our Kids Study. Hypertension 53(4): 611-616.

31. Zieman SJ, Melenovsky V, Kass DA (2005) Mechanisms, Pathophysiology, and therapy of arterial stiffness. Arterioscler Thromb Vasc Biol 25(5): 932-943.

32. Nichols WW (2005) Clinical measurement of arterial stiffness obtained from noninvasive pressure waveforms. Am J Hypertens 18(1-2): 3-10.

33. Topouchian J, Labat C, Gautier S, Benetos A, Asmar R, et al. (2018) Effects of metabolic syndrome on arterial function in different age groups: The Advanced Approach to Arterial Stiffness study. J Hypertens 36(4): 824833.

34. Mancia G, Bousquet P, Elghozi JL (2007) The sympathetic nervous system and the metabolic syndrome. Journal of hypertension 25(5): 909-920.

35. Garnier AS, Briet M (2015) Arterial stiffness and chronic kidney disease. Pulse 363(4): 229-241. 